Research Paper

\title{
Albumin-to-Alkaline Phosphatase Ratio is an Independent Prognostic Indicator in Combined Hepatocellular and Cholangiocarcinoma
}

\author{
Feng Zhang, Shenxin Lu, Mengxin Tian, Keshu Hu, Rongxin Chen, Boheng Zhang, Zhenggang Ren, \\ Yinghong Shi ${ }^{\bowtie}$ and Xin Yin \\ Liver Cancer Institute \& Zhongshan Hospital, Fudan University, Shanghai, China; Key Laboratory of Carcinogenesis and Cancer Invasion, Ministry of \\ Education.
}

$\triangle$ Corresponding authors: Xin Yin, Liver Cancer Institute \& Zhongshan Hospital, Fudan University, 136 Yi Xue Yuan Road, Shanghai 200032, China. Tel.: 86-21-64038038; Fax: 86-21-64038038; E-mail: yin.xin@zs-hospital.sh.cn. Yinghong Shi, Liver Cancer Institute \& Zhongshan Hospital, Fudan University, 136 Yi Xue Yuan Road, Shanghai 200032, China. Tel.: 86-21-64038038; Fax: 86-21-64038038; E-mail: shi.yinghong@zs-hospital.sh.cn.

(C) The author(s). This is an open access article distributed under the terms of the Creative Commons Attribution License (https://creativecommons.org/licenses/by/4.0/). See http://ivyspring.com/terms for full terms and conditions.

Received: 2020.03.04; Accepted: 2020.06.12; Published: 2020.06.29

\begin{abstract}
Background: The clinical significance of Albumin-to-Alkaline Phosphatase Ratio (AAPR) has been discussed in hepatocellular carcinoma (HCC) and cholangiocarcinoma (CC). The aim of this study is to clarify the prognostic value of AAPR in patients with combined hepatocellular and cholangiocarcinoma (cHCC-CCA).

Methods: A total of 267 patients pathologically diagnosed as Allen type $\mathrm{C}$ cHCC-CCA in our institution were retrospectively enrolled and randomly divided into the training $(\mathrm{N}=187)$ cohort and validation $(\mathrm{N}=80)$ cohort. The prognostic value of AAPR was evaluated and validated. An AAPR-based nomogram was constructed and its prediction performance was assessed.

Results: We identified 0.43 as the optimal threshold value of AAPR by the $X$-tile software. In the training cohort, the median overall survival (OS) of patients with AAPR $<0.43$ was significant shorter than that of those with $A A P R \geq 0.43$ ( 15.8 months vs 35 months, respectively, $P<0.001$ ). Univariate and multivariate analyses demonstrated that AAPR was a strong indicator of OS. The concordance index (C-index), receiver operating characteristic (ROC) curves, likelihood ratio tests (LAT), Akaike information criteria (AIC) and decision curve analysis (DCA) demonstrated that AAPR outperformed the Child-Pugh (CP) grade and albumin-bilirubin (ALBI) grade in predicting $O S$. These findings were further verified in the validation cohort. The AAPR-based nomogram achieved C-index values of 0.76 ( $95 \% \mathrm{Cl}: 0.71-0.81)$ in the training cohort and $0.69(95 \% \mathrm{Cl}: 0.60-0.78)$ in the validation cohort, which presented significant superiority to TNM stage.

Conclusions: Preoperative AAPR is an independent prognostic predictor in cHCC-CCA. The AAPR-based nomogram contributes to personalized prognosis prediction and clinical decision making for cHCC-CCA.
\end{abstract}

Key words: Albumin-to-Alkaline Phosphatase Ratio; combined hepatocellular and cholangiocarcinoma; overall survival; serum biomarker

\section{Introduction}

Combined hepatocellular-cholangiocarcinoma (cHCC-CCA) is a rare primary liver cancer showing clinical and pathological features of both hepatocellular carcinoma (HCC) and cholangiocarcinoma (CC). The incidence rate of this tumor was reported to be $0.4 \%-14.2 \%$ in different regions [1]. Recent studies indicated that cHCC-CCA may derived from the clonal hepatic progenitor cells (HPCs) with bipotential differentiation into either hepatocytic and cholangiocytic lineages [2]. In 1949, Allen and Lisa 
classified cHCC-CCA into three subtypes (A: double type; B: combined type; C: mixed type) [3]. However, the latest 2019 WHO classification omits the subcategorization and solely defines the cHCC-CCA as the subtype with unequivocal components of both HCC and CC intimately mixed in the same mass, which was previously described as type $\mathrm{C}$ neoplasm by Allen and Lisa [4].

The prognosis of cHCC-CCA has been explored in previous studies. Some researchers suggested that cHCC-CCA had poorer survival outcome than both HCC and CC [5], whereas other investigations showed a better or similar prognosis of cHCC-CCA compared to CC $[6,7]$. Several prognostic factors for cHCC-CCA have been identified, including performance status, Child-Pugh $(\mathrm{CP})$ grade, tumor size, tumor number, carcinoembryonic antigen (CEA) and carbohydrate antigen 19-9 (CA19-9) [7-10]. However, these findings still remain controversial and need further validations, due to the small sample size and inconsistent pathological diagnostic criteria.

Albumin-to-Alkaline Phosphatase Ratio (AARP) is a newly developed serum biomarker-based index, which is calculated as albumin (ALB) level divided by alkaline phosphatase (AKP) level. The prognostic value of AAPR has been clarified in several malignancies including HCC [11, 12], CC [13], pancreatic ductal adenocarcinoma [14] and lung cancer [15]. With regard to liver cancer, recent studies indicated that AAPR might not only reflect liver function reserve, but also associate with inflammation and cancer cell proliferation [12]. However, no research has ever evaluated the prognostic value of AAPR in patients with CHCC-CCA.

Therefore, in this study, we for the first time explored the prediction performance of preoperative AAPR in patients with cHCC-CCA. Moreover, an AAPR-based nomogram was constructed to facilitate individualized prognosis prediction and clinical decision making.

\section{Materials and Methods}

\section{Patients}

We retrospectively screened 267 cHCC-CCA patients treated in liver cancer institute, Zhongshan Hospital between January 1993 and December 2015. The inclusion criteria were as follows: 1) pathologically confirmed as Allen type $\mathrm{C}$ cHCC-CCA by liver resection; 2 ) with $\mathrm{CP}$ grade $\mathrm{A}$ or $\mathrm{B} ; 3$ ) with detailed preoperative laboratory data and integrated follow-up data. The exclusion criteria were as follows: 1) with synchronous or previous malignancies; 2) with known renal or bone diseases. Subsequently, 267 cHCC-CCA patients were randomly divided into the training cohort $(\mathrm{N}=187)$ and validation cohort $(\mathrm{N}=80)$ in a 7:3 ratio.

The baseline and clinical characteristics of patients were collected, including age, sex, etiology, presence of underlying liver cirrhosis, laboratory results and tumor-related characteristics. The TNM stage was defined according to the eighth edition of the TNM staging system [16]. The AARP was calculated by dividing the serum ALB ( $\mathrm{g} / \mathrm{L}$ ) by serum AKP (U/L). The overall survival (OS) time was defined as the interval between the treatment date and the death date or the last follow-up date.

\section{Treatment and Follow-up}

The surgical procedure has been described elsewhere [17]. All patients were followed up every 2-3 months within the first year after hepatic resection, and thereafter, every 6 months. Physical examinations, routine blood tests, liver function tests, tumor maker tests were practiced routinely. Radiological examinations including chest radiography, computerized tomography (CT) or magnetic resonance imaging (MRI) were performed to screen tumor recurrence.

\section{Statistical Analysis}

Continuity variables were expressed as mean with standard deviation (SD) and compared by student's $t$ test. Categorical variables were described as number with percentages and compared by either Pearson $\chi^{2}$ analysis or Fisher's exact test.

The optimal threshold value of AAPR was determined by the X-tile statistical software (version 3.6.1, Yale University, New Haven, CT, USA) [18] regarding to OS. Patients were divided into either the low-AAPR or high-AAPR group according to the defined cut-off value. Kaplan-Meier analysis was employed to estimate the survival curves and log-rank test was used to examine the survival differences. Univariate analysis was performed to determine significant variables associated with OS. Variables with $P$ value $<0.1$ were further included in multivariate Cox proportional hazards regression model. The predictive performances of AAPR, CP grade and albumin-bilirubin (ALBI) grade were compared by using the concordance index (C-index) values, area under the curves (AUCs), likelihood ratio tests (LAT) and Akaike information criteria (AIC). In addition, decision curve analysis (DCA) was performed evaluating the net benefit of different liver function indices by using $\mathrm{R}$ package rmda [19]. The AAPR-based nomogram for predicting 1-, 2- and 3 -year OS rates was constructed based on independent prognostic factors identified by multivariate analysis. The calibration plots, C-index, 
AUCs, LAT and AIC were employed to assess the predictive value of the model in comparison with the 8th edition of American Joint Committee on Cancer TNM staging (AJCC TNM-8).

All statistical analyses were performed by using Stata software (version 15.1, StataCorp, College station, TX) and R software (version 3.5.1). A twotailed $\mathrm{P}$ value of less than 0.05 was considered as statistically significant.

\section{Ethical Statement}

This study was approved by Medical Ethics Committee of Zhongshan Hospital affiliated to Fudan University (No: B2019-169) and complies with the Helsinki Declaration.

\section{Results}

\section{Baseline clinical characteristics}

The main demographic and clinical characteristics of the training cohort and validation cohort were shown in Table 1 . In the training cohort, there were $146(78.1 \%)$ male and $41(21.9 \%)$ female patients, with a mean age of $52.6 \pm 11.7$ years. 141 (75.4\%) patients had a background of hepatitis B virus (HBV) infection. 131 (70.1\%) patients had liver cirrhosis and $156(83.4 \%)$ patients exhibited CP grade A. As for laboratory results, the mean level of ALB and AKP were $41.4 \pm 4.3 \mathrm{~g} / \mathrm{L}$ and $93.9 \pm 37.8 \mathrm{U} / \mathrm{L}$, respectively. And Alfa-Fetoprotein (AFP), CEA and CA19-9 were positive in 103 (55.1\%), 29 (15.5\%) and 59 $(31.6 \%)$ patients, respectively. As for the tumor characteristics, there were $96(51.3 \%)$ patients with tumor diameter larger than $5 \mathrm{~cm}$ and 64 (34.2\%) patients with multiple tumors. Macroscopic vascular invasion (MVI) and lymph node involvement (LNI) were detected in $17(9.1 \%)$ and 18 (9.6\%) patients, respectively. The number of patients classified as AJCC-TNM I, II, III and IV were 83 (44.4\%), 35 (18.7\%), $47(25.1 \%)$ and $22(11.8 \%)$, respectively.

\section{Clinicopathological characteristics of patients in the low-AAPR group and high-AAPR group}

The optimal threshold value of AAPR was determined as 0.43 based on X-tile analyses in the training cohort (Figure S1). Subsequently, patients were dichotomized into either low-AAPR group (AAPR $<0.43, \mathrm{~N}=75$ ) or high-AAPR group (AAPR $\geq$ $0.43, \mathrm{~N}=112$ ). The correlations between AAPR and other clinicopathological features were shown in Table 2. Patients with low-AAPR levels had older age, poorer liver function (CP grade B or ALBI grade $2 \& 3$ ) and higher levels of $\gamma$-glutamyl transpeptidase $(\gamma-G T)$, CEA and CA19-9 (P < 0.05). No significant associations were found between AAPR and gender, etiology, liver cirrhosis, alanine aminotransferase
(ALT), total bilirubin (TB), AFP, tumor characteristics and TNM stage.

Table 1. Characteristics of the training and validation cohorts

\begin{tabular}{|c|c|c|c|c|}
\hline Characteristics & $\begin{array}{l}\text { Total } \\
(\mathrm{N}=267)\end{array}$ & $\begin{array}{l}\text { Training } \\
\text { cohort }(\mathrm{N}=187)\end{array}$ & $\begin{array}{l}\text { Validation cohort } \\
(\mathrm{N}=80)\end{array}$ & $\begin{array}{l}\mathrm{P} \\
\text { value }\end{array}$ \\
\hline Age (years) & $52.3 \pm 11.6$ & $52.6 \pm 11.7$ & $51.5 \pm 11.3$ & 0.466 \\
\hline Sex & & & & 0.326 \\
\hline Male & 204 (76.4) & $146(78.1)$ & $58(72.5)$ & \\
\hline Female & $63(23.6)$ & 41 (21.9) & $22(27.5)$ & \\
\hline Etiology & & & & 0.515 \\
\hline HBV infection & $196(73.4)$ & $141(75.4)$ & $55(68.8)$ & \\
\hline $\mathrm{HCV}$ infection & $5(1.9)$ & $3(1.6)$ & $2(2.5)$ & \\
\hline Other & $66(24.7)$ & $43(23.0)$ & $23(28.8)$ & \\
\hline $\begin{array}{l}\text { Liver cirrhosis } \\
\text { (yes) }\end{array}$ & $186(69.7)$ & $131(70.1)$ & $55(68.8)$ & 0.832 \\
\hline ALT (U/L) & $44.6 \pm 56.4$ & $41.4 \pm 48.5$ & $52.0 \pm 71.5$ & 0.160 \\
\hline $\operatorname{ALB}(g / L)$ & $41.4 \pm 4.5$ & $41.4 \pm 4.3$ & $41.5 \pm 4.9$ & 0.881 \\
\hline TB (umol/L) & $15.7 \pm 24.0$ & $13.8 \pm 14.3$ & $20.0 \pm 37.7$ & 0.053 \\
\hline AKP (U/L) & $101.6 \pm 89.4$ & $93.9 \pm 37.8$ & $119.7 \pm 151.8$ & 0.030 \\
\hline ү-GT (U/L) & & & & 0.432 \\
\hline$\leq 50$ & 103 (38.6) & 75 (40.1) & $28(35.0)$ & \\
\hline$>50$ & $164(61.4)$ & 112 (59.9) & $52(65.0)$ & \\
\hline AARP & $0.50 \pm 0.22$ & $0.51 \pm 0.21$ & $0.47 \pm 0.25$ & 0.264 \\
\hline CP grade & & & & 0.667 \\
\hline A & $221(82.8)$ & $156(83.4)$ & $65(81.3)$ & \\
\hline B & $46(17.2)$ & 31 (16.6) & $15(18.8)$ & \\
\hline ALBI grade & & & & 0.213 \\
\hline Grade 1 & $148(55.4)$ & $108(57.8)$ & $40(50.0)$ & \\
\hline Grade 2 & $114(42.7)$ & 77 (41.2) & 37 (46.3) & \\
\hline Grade 3 & $5(1.9)$ & $2(1.1)$ & $3(3.8)$ & \\
\hline $\operatorname{AFP}(\mathrm{ng} / \mathrm{mL})$ & & & & 0.841 \\
\hline$\leq 20$ & $121(45.3)$ & $84(44.9)$ & 37 (46.3) & \\
\hline$>20$ & $146(54.7)$ & $103(55.1)$ & $43(53.8)$ & \\
\hline CEA(ug/L) & & & & 0.369 \\
\hline$\leq 5$ & $222(83.2)$ & $158(84.5)$ & $64(80.0)$ & \\
\hline$>5$ & 45 (16.9) & $29(15.5)$ & $16(20.0)$ & \\
\hline CA19-9(ku/L) & & & & 0.649 \\
\hline$\leq 37$ & 185 (69.3) & $128(68.5)$ & $57(71.3)$ & \\
\hline$>37$ & $82(30.7)$ & 59 (31.6) & $23(28.8)$ & \\
\hline Tumor size $(\mathrm{cm})$ & & & & 0.256 \\
\hline$\leq 5$ & $136(50.9)$ & $91(48.7)$ & $45(56.3)$ & \\
\hline$<5$ & $131(49.1)$ & $96(51.3)$ & $35(43.8)$ & \\
\hline Tumor number & & & & 0.200 \\
\hline Single & $182(68.2)$ & $123(65.8)$ & $59(73.8)$ & \\
\hline Multiple & $85(31.8)$ & $64(34.2)$ & $21(26.3)$ & \\
\hline MVI & & & & 0.129 \\
\hline Absent & 247 (92.5) & $170(90.9)$ & 77 (96.3) & \\
\hline Present & $20(7.5)$ & $17(9.1)$ & $3(3.8)$ & \\
\hline LNI & & & & 0.483 \\
\hline Absent & $239(89.5)$ & $169(90.4)$ & $70(87.5)$ & \\
\hline Present & $28(10.5)$ & $18(9.6)$ & $10(12.5)$ & \\
\hline AJCC TNM-8 & & & & 0.355 \\
\hline I & $117(43.8)$ & $83(44.4)$ & $34(42.5)$ & \\
\hline II & $53(19.9)$ & $35(18.7)$ & $18(22.5)$ & \\
\hline III & $61(22.9)$ & $47(25.1)$ & $14(17.5)$ & \\
\hline IV & $36(13.5)$ & $22(11.8)$ & $14(17.5)$ & \\
\hline
\end{tabular}

Note: HBV: hepatitis B virus; HCV: hepatitis C virus; ALT: alanine aminotransferase; ALB: albumin; TB: total bilirubin; AKP: alkaline phosphatase; $\gamma$-GT: $\gamma$-glutamyl transpeptidase; AAPR: Albumin-to-Alkaline Phosphatase Ratio; CP grade: Child-Pugh grade; ALBI grade: the albumin-bilirubin grade; AFP: Alfa-Fetoprotein; CEA: carcinoembryonic antigen; CA19-9: carbohydrate antigen 19-9; MVI: macroscopic vascular invasion; LNI: lymph node involvement; AJCC TNM-8: the 8th edition of American Joint Committee on Cancer TNM staging. 
Table 2. Association between AAPR and other characteristics

\begin{tabular}{|c|c|c|c|c|}
\hline Characteristics & $\begin{array}{l}\text { Total } \\
(\mathrm{N}=187)\end{array}$ & $\begin{array}{l}\text { AAPR } \geq 0.43 \\
(\mathrm{~N}=112)\end{array}$ & $\begin{array}{l}\text { AAPR }<0.43 \\
(\mathrm{~N}=75)\end{array}$ & $P$ value \\
\hline Age (years) & $52.6 \pm 11.7$ & $51.0 \pm 11.2$ & $55.1 \pm 12.2$ & $0.019^{*}$ \\
\hline Sex & & & & 0.100 \\
\hline Male & $146(78.1)$ & $92(82.1)$ & $54(72.0)$ & \\
\hline Female & $41(21.9)$ & $20(17.9)$ & $21(28.0)$ & \\
\hline Etiology & & & & 0.183 \\
\hline HBV infection & $141(75.4)$ & $90(80.4)$ & $51(68.0)$ & \\
\hline $\mathrm{HCV}$ infection & $3(1.6)$ & $\backslash$ & $3(4.0)$ & \\
\hline Other & $43(23.0)$ & $22(19.6)$ & $21(28.0)$ & \\
\hline Liver cirrhosis (yes) & $131(70.1)$ & $78(69.6)$ & $53(70.7)$ & 0.881 \\
\hline ALT (U/L) & $41.4 \pm 48.5$ & $36.5 \pm 35.1$ & $48.8 \pm 63.0$ & 0.089 \\
\hline $\operatorname{ALB}(g / L)$ & $41.4 \pm 4.3$ & $42.5 \pm 4.1$ & $39.7 \pm 4.2$ & $<0.001^{*}$ \\
\hline TB (umol/L) & $13.8 \pm 14.3$ & $12.2 \pm 5.5$ & $16.1 \pm 21.5$ & 0.068 \\
\hline $\mathrm{AKP}(\mathrm{U} / \mathrm{L})$ & $93.9 \pm 37.8$ & $73.1 \pm 15.7$ & $124.8 \pm 40.1$ & $<0.001^{*}$ \\
\hline Y-GT (U/L) & & & & $<0.001^{*}$ \\
\hline$\leq 50$ & $75(40.1)$ & $59(52.7)$ & $16(21.3)$ & \\
\hline$>50$ & $112(59.9)$ & $53(47.3)$ & $59(78.7)$ & \\
\hline AARP & $0.51 \pm 0.21$ & $0.62 \pm 0.20$ & $0.34 \pm 0.07$ & $<0.001^{*}$ \\
\hline CP grade & & & & $0.001^{*}$ \\
\hline $\mathrm{A}$ & $156(83.4)$ & $102(91.1)$ & $54(72.0)$ & \\
\hline B & $31(16.6)$ & $10(8.9)$ & $21(28.0)$ & \\
\hline ALBI grade & & & & $<0.001^{*}$ \\
\hline Grade 1 & $108(57.8)$ & $78(69.6)$ & $30(40.0)$ & \\
\hline Grade 2 & $77(41.2)$ & $34(30.4)$ & $43(57.3)$ & \\
\hline Grade 3 & $2(1.1)$ & 1 & $2(2.7)$ & \\
\hline $\operatorname{AFP}(\mathrm{ng} / \mathrm{mL})$ & & & & 0.694 \\
\hline$\leq 20$ & $84(44.9)$ & $49(43.8)$ & $35(46.7)$ & \\
\hline$>20$ & $103(55.1)$ & $63(56.3)$ & $40(53.3)$ & \\
\hline CEA (ug/L) & & & & $0.027^{*}$ \\
\hline$\leq 5$ & $158(84.5)$ & $100(89.3)$ & $58(77.3)$ & \\
\hline$>5$ & $29(15.5)$ & $12(10.7)$ & $17(22.7)$ & \\
\hline CA19-9 (ku/L) & & & & $0.018^{*}$ \\
\hline$\leq 37$ & $128(68.5)$ & $84(75.0)$ & $44(58.7)$ & \\
\hline$>37$ & $59(31.6)$ & $28(25.0)$ & $31(41.3)$ & \\
\hline Tumor size $(\mathrm{cm})$ & & & & 0.052 \\
\hline$\leq 5$ & $91(48.7)$ & $61(54.5)$ & $30(40.0)$ & \\
\hline$<5$ & $96(51.3)$ & $51(45.5)$ & $45(60.0)$ & \\
\hline Tumor number & & & & 0.675 \\
\hline Single & $123(65.8)$ & $75(67.0)$ & $48(64.0)$ & \\
\hline Multiple & $64(34.2)$ & $37(33.0)$ & $27(36.0)$ & \\
\hline AJCC TNM-8 & & & & 0.066 \\
\hline I & $83(44.4)$ & $54(48.2)$ & $29(38.7)$ & \\
\hline II & $35(18.7)$ & $24(21.4)$ & $11(14.7)$ & \\
\hline III & $47(25.1)$ & $23(20.5)$ & $24(32.0)$ & \\
\hline IV & $22(11.8)$ & $11(9.8)$ & $11(14.7)$ & \\
\hline MVI & & & & 0.122 \\
\hline Absent & $170(90.9)$ & $105(93.8)$ & $65(86.7)$ & \\
\hline Present & $17(9.1)$ & $7(6.3)$ & $10(13.3)$ & \\
\hline LNI & & & & 0.450 \\
\hline Absent & $169(90.4)$ & $103(92.0)$ & $66(88.0)$ & \\
\hline Present & $18(9.6)$ & $9(8.0)$ & $9(12.0)$ & \\
\hline
\end{tabular}

Note: HBV: hepatitis B virus; HCV: hepatitis C virus; ALT: alanine

aminotransferase; ALB: albumin; TB: total bilirubin; AKP: alkaline phosphatase; Y-GT: Y-glutamyl transpeptidase; AAPR: Albumin-to-Alkaline Phosphatase Ratio; CP grade: Child-Pugh grade; ALBI grade: the albumin-bilirubin grade; AFP: Alfa-Fetoprotein; CEA: carcinoembryonic antigen; CA19-9: carbohydrate antigen 19-9; MVI: macroscopic vascular invasion; LNI: lymph node involvement; AJCC TNM-8: the 8th edition of American Joint Committee on Cancer TNM staging.

\section{Survival analyses}

The mean follow-up duration was 25.2 (Range: 1-227) months. At the end of follow-up, 147 (55.1\%) patients died and $112(42.0 \%)$ patients showed tumor recurrence. In the training cohort, the median OS time was 29 (95\%CI: 18-36) months and the 1-, 2- and 3 -year OS rates were $70.8 \%, 52.0 \%$ and $40.1 \%$, respectively. In the validation cohort, the median OS time was 24 (95\%CI: 18-36) months and the 1-, 2- and 3 -year OS rates were $79.3 \%, 48.1 \%$ and $36.6 \%$, respectively. No significant survival differences were found between the training cohort and validation cohort $(\mathrm{P}=0.972)$.

As shown in Figure 1, patients with low AAPR levels presented significant poorer survival compared to patients with high AAPR levels. In the training cohort, the median OS time of patients with low AAPR and high AAPR levels were 15.8 months vs 35 months, respectively. And the 1-, 2- and 3-year OS rates were $70.8 \%, 52.0 \%, 40.1 \%$ in the low-AAPR group, $79.3 \%, 48.1 \%, 36.6 \%$ in the high-AAPR group, respectively (Figure 1A). Furthermore, these findings were also verified in the validation cohort (Figure 1B).

\section{Univariate and multivariate analyses}

In the training cohort, univariate analysis suggested AAPR, CP grade, $\mathrm{y}$-GT, CEA, CA19-9, tumor size, tumor number, MVI, LNI and TNM stage were significantly associated with OS. By further multivariate analysis, AAPR, Y-GT, CEA, CA19-9 and TNM stage were identified as significant independent prognostic factors. While in the validation cohort, univariate and multivariate analyses confirmed that AAPR and TNM stage were strong indicators of OS in patients with cHCC-CCA (Table 3).

\section{Prognostic prediction performance of AAPR in comparison with different liver function assessment methods}

The prognostic prediction performances of AAPR, CP grade and ALBI grade were evaluated by using C-index, AUCs, LRT and AIC (Table 4, Figure 2). In the training cohort, the C-index values of AAPR, CP grade and ALBI grade were 0.61 (95\% CI: $0.55-0.67), 0.57$ (95\%CI: 0.52-0.62) and 0.56 (95\%CI: $0.50-0.62)$, respectively. The AUCs of AAPR, CP grade and ALBI grade were 0.63 (95\%CI: 0.56-0.70), 0.59 (95\%CI: $0.52-0.66)$ and 0.55 (95\%CI: $0.50-0.60)$, respectively. In addition, AAPR had the largest LAT $\chi^{2}$ of 11.7 and lowest AIC value of 854, which outperformed CP grade and ALBI grade. In the validation cohort, AAPR also displayed the largest C-index value, AUCs, LAT $\chi^{2}$ value and lowest AIC value. Furthermore, DCA demonstrated that AAPR had superior clinical usefulness compared to CP grade and ALBI grade (Figure 3). All these findings suggested AAPR could serve as a better prognostic predictor in comparison with $\mathrm{CP}$ grade and ALBI grade in $\mathrm{CHCC}-\mathrm{CCA}$. 


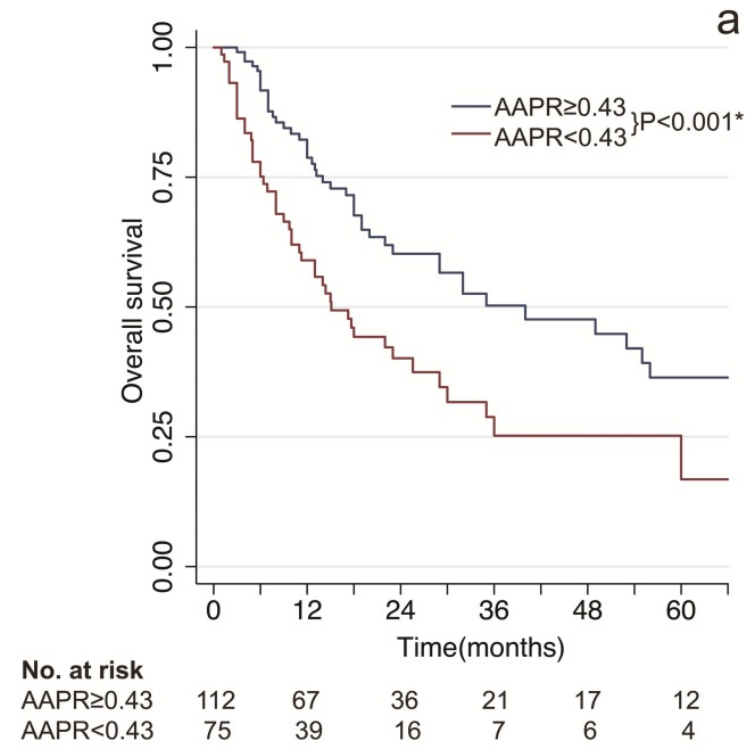

a

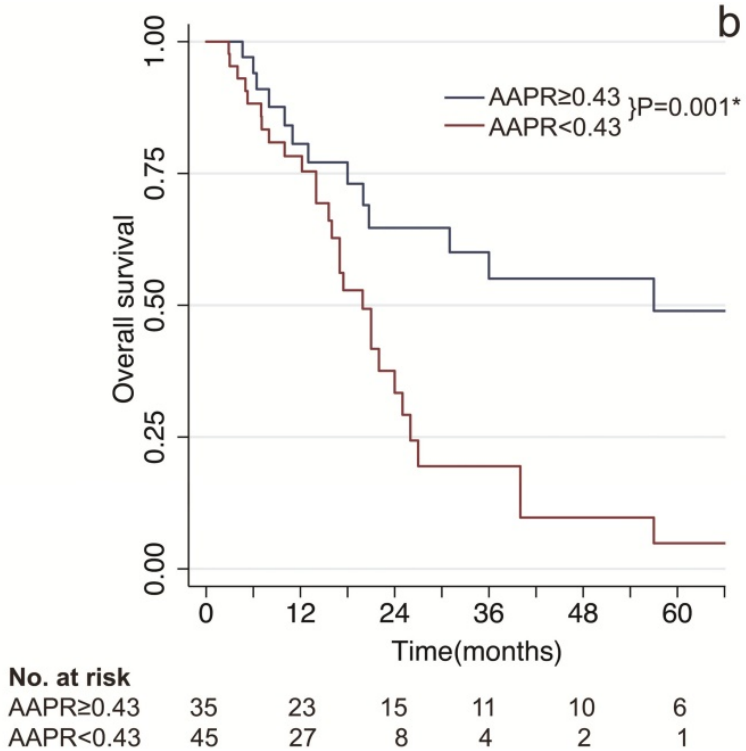

Figure 1. Kaplan-Meier survival estimates between subgroups by AAPR. (a) overall survival by AAPR in the training cohort; (b) overall survival by AAPR in the validation cohort. AAPR: albumin-to-alkaline phosphatase ratio. ${ }^{*}$ statistically significant.

Table 3. Univariate and multivariate analyses for overall survival in the training cohort and validation cohort

\begin{tabular}{|c|c|c|c|c|c|c|}
\hline \multirow[t]{3}{*}{ Variable } & \multicolumn{3}{|l|}{ Training cohort } & \multicolumn{3}{|l|}{ Validation cohort } \\
\hline & \multirow{2}{*}{$\begin{array}{l}\text { Univariate analysis } \\
\text { p-value }\end{array}$} & \multicolumn{2}{|c|}{ Multivariate analysis } & \multirow{2}{*}{$\begin{array}{l}\text { Univariate analysis } \\
\text { p-value }\end{array}$} & \multicolumn{2}{|c|}{ Multivariate analysis } \\
\hline & & HR $(95 \% \mathrm{CI})$ & p-value & & HR $(95 \% \mathrm{CI})$ & p-value \\
\hline Age, years $(\leq 65 />65)$ & 0.091 & & & 0.768 & & \\
\hline Sex (female/male) & 0.285 & & & 0.789 & & \\
\hline Hepatitis (no/yes) & 0.651 & & & 0.337 & & \\
\hline Liver cirrhosis (no/yes) & 0.052 & & & 0.510 & & \\
\hline ALT, U/L $(\leq 40 />40)$ & 0.057 & & & 0.821 & & \\
\hline$\gamma-\mathrm{GT}, \mathrm{U} / \mathrm{L}(\leq 50 />50)$ & $<0.001^{*}$ & $2.36(1.44-3.85)$ & $0.001^{*}$ & $0.012^{*}$ & & \\
\hline AARP $(>0.40 / \leq 0.40)$ & $<0.001^{*}$ & $1.77(1.16-2.68)$ & $0.007^{*}$ & $0.001^{*}$ & $2.19(1.06-4.51)$ & $0.034^{*}$ \\
\hline $\mathrm{CP}$ grade $(\mathrm{A} / \mathrm{B})$ & $0.020^{*}$ & & & 0.619 & & \\
\hline ALBI grade $(1 / 2 / 3)$ & 0.120 & & & 0.432 & & \\
\hline $\mathrm{AFP}, \mathrm{ng} / \mathrm{mL}(\leq 400 />400)$ & 0.386 & & & 0.466 & & \\
\hline CEA, ug/L $(\leq 5 />5)$ & $0.008^{*}$ & $1.69(1.03-2.76)$ & $0.036^{*}$ & 0.073 & & \\
\hline CA19-9, ku/L $(\leq 37 />37)$ & $<0.001^{*}$ & $1.65(1.08-2.54)$ & $0.022^{*}$ & 0.056 & & \\
\hline Tumor size, $\mathrm{cm}(\leq 5 />5)$ & $<0.001^{*}$ & & & $<0.001^{*}$ & & \\
\hline Tumor number (single / multiple) & $0.020^{*}$ & & & $0.014^{*}$ & & \\
\hline MVI (no/yes) & $<0.001^{*}$ & & & 0.147 & & \\
\hline LNI (no/yes) & $<0.001^{*}$ & & & $0.028^{*}$ & & \\
\hline AJCC TNM-8 (I/II/III/IV) & $<0.001^{*}$ & $1.75(1.43-2.14)$ & $<0.001^{*}$ & $<0.001^{*}$ & $1.77(1.32-2.36)$ & $<0.001^{*}$ \\
\hline
\end{tabular}

Note: HR: hazard ratio; CI: confidence interval; ALT: alanine aminotransferase; $\gamma$-GT: $\gamma$-glutamyl transpeptidase; AAPR: Albumin-to-Alkaline Phosphatase Ratio; CP grade: Child-Pugh grade; ALBI grade: the albumin-bilirubin grade; AFP: Alfa-Fetoprotein; CEA: carcinoembryonic antigen; CA19-9: carbohydrate antigen 19-9; MVI: macroscopic vascular invasion; LNI: lymph node involvement; AJCC TNM-8: the 8th edition of American Joint Committee on Cancer TNM staging. *statistically significant.

\section{The AAPR-based nomogram and its predictive value}

In the training cohort, by using multivariate Cox proportional hazards model, 5 variables were identified as independent prognostic factors, including AAPR, $y$-GT, CEA, CA19-9 and TNM stage. The nomogram which predicted 1-, 2- and 3-year OS was constructed based on the 5 variables and each variable was assigned a score according to their $\beta$ coefficients (Figure 4). The calibration plots in both training and validation cohorts proved the excellent predictive value of the AAPR-based nomogram
(Figure 5). Moreover, the AAPR-based prognostic nomogram significantly outperformed TNM stage by using C-index, AUCs, LRT and AIC in both training and validation cohorts (Table 5).

\section{Discussion}

cHCC-CCA is a distinct form of primary liver cancer showing features of both HCC and CC [20]. Due to the low incidence of CHCC-CCA, there is still a lack of investigation on prognostic prediction in cHCC-CCA. In present study, we for the first time identified the prognostic value of AAPR in patients with cHCC-CCA. 
Table 4. Comparison of the predictive performances of liver function assessment methods

\begin{tabular}{|c|c|c|c|c|c|c|c|c|}
\hline \multirow[t]{2}{*}{ Model } & \multicolumn{4}{|l|}{ Training cohort } & \multicolumn{4}{|l|}{ Validation cohort } \\
\hline & C-index $(95 \% \mathrm{CI})$ & AUC (95\%CI) & LAT $\chi^{2}$ & AIC & C-index $(95 \% \mathrm{CI})$ & AUC (95\%CI) & LAT $\chi^{2}$ & AIC \\
\hline AAPR & $0.61(0.55-0.67)$ & $0.63(0.56-0.70)$ & 11.7 & 854 & $0.59(0.51-0.67)$ & $0.62(0.51-0.73)$ & 10.8 & 297 \\
\hline $\mathrm{CP}$ grade & $0.57(0.52-0.62)$ & $0.55(0.50-0.60)$ & 4.6 & 861 & $0.48(0.43-0.53)$ & $0.50(0.42-0.59)$ & 0.2 & 308 \\
\hline ALBI grade & $0.56(0.50-0.62)$ & $0.59(0.52-0.66)$ & 4.0 & 861 & $0.53(0.45-0.61)$ & $0.47(0.35-0.58)$ & 1.3 & 307 \\
\hline
\end{tabular}

Note: AUC: area under the curve; C-index: concordance index; LAT $\chi 2$ : likelihood ratio test $\chi^{2}$; AIC: Akaike information criteria; CI: confidence interval; AAPR:

albumin-to-alkaline phosphatase ratio; CP grade: Child-Pugh grade; ALBI grade: the albumin-bilirubin grade.

a

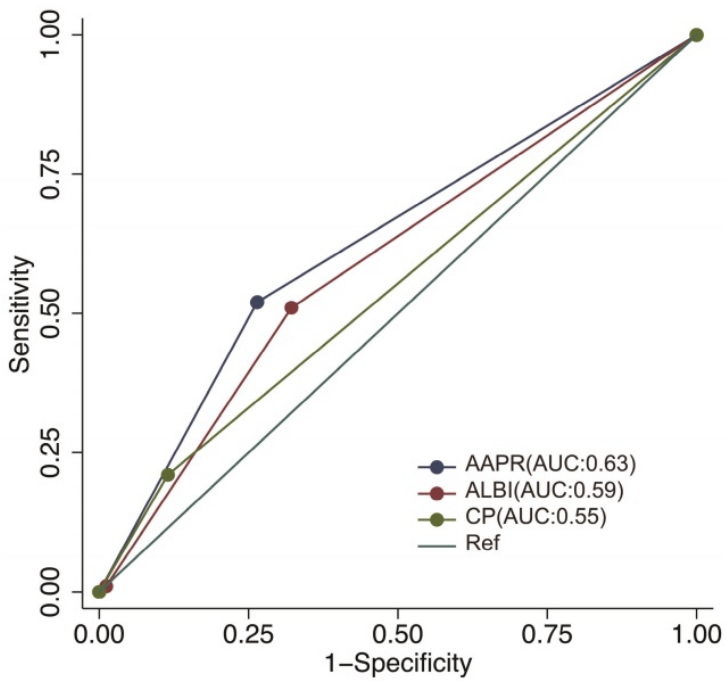

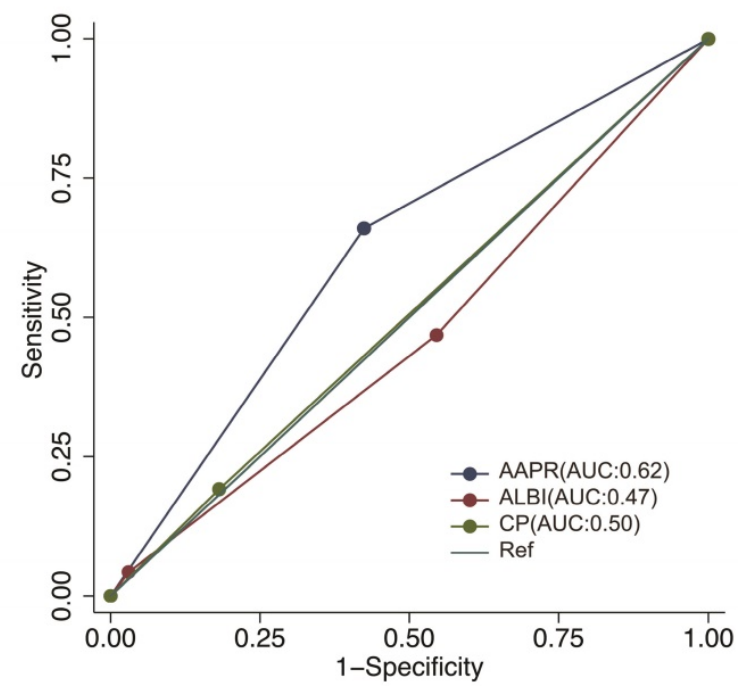

Figure 2. The ROC curves of $A A P R, C P$ grade and $A L B I$ grade for predicting overall survival in the training cohort and validation cohort. (a) ROC curves in the training cohort; (b) ROC curves in the validation cohort. ROC, receiver operating characteristic; AAPR: albumin-to-alkaline phosphatase ratio; CP grade: Child-Pugh grade; ALBI grade: albumin-bilirubin grade.

a
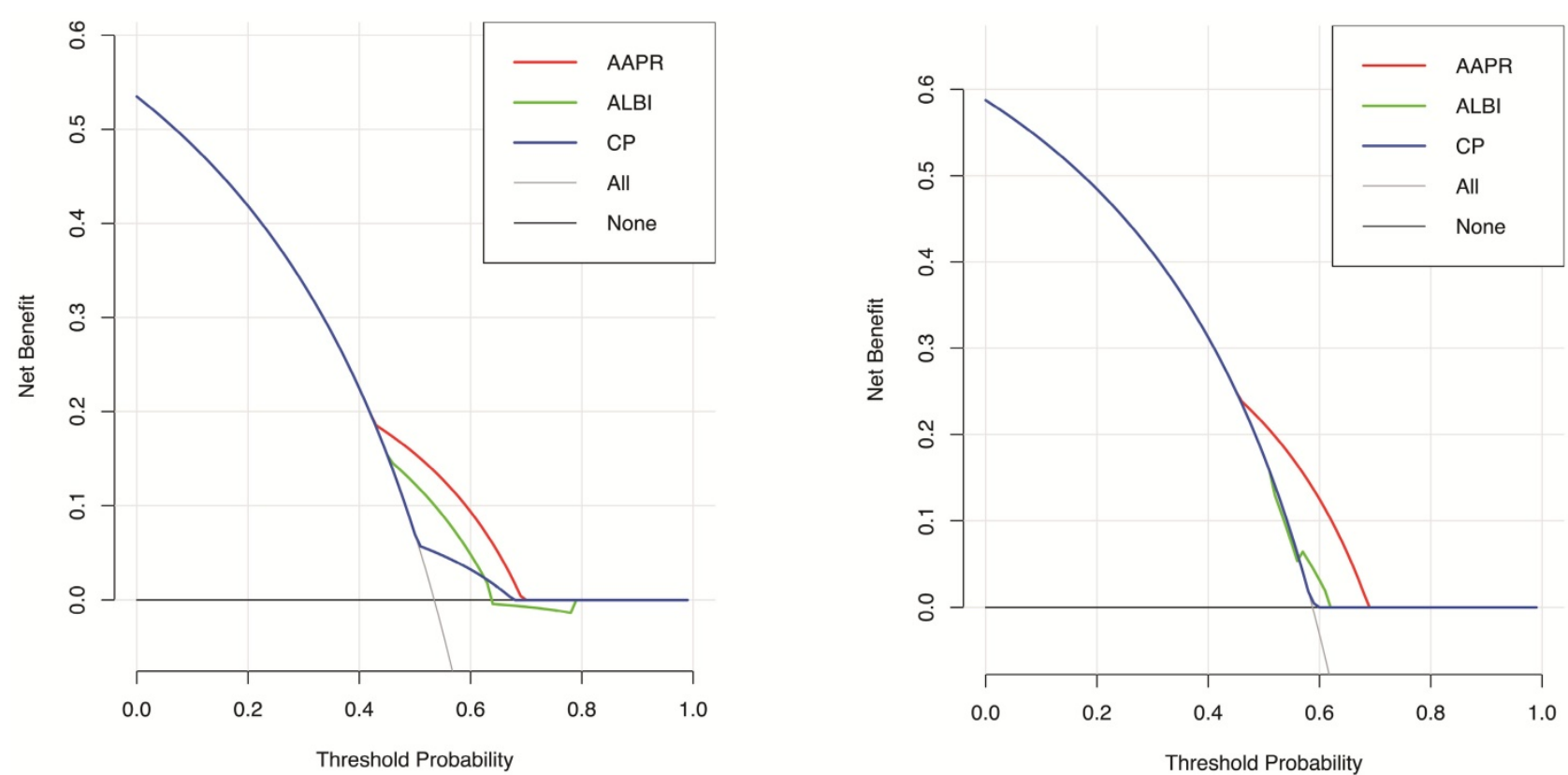

Figure 3. DCA for OS of different liver function assessment methods. (a) DCA for OS in the training cohort. (b) DCA for OS in the validation cohort. DCA: decision curve analysis; OS: overall survival. 
Points

$10 \quad 20$

30

$40 \quad 50$

$50 \quad 60$

70

AAPR

$\mathrm{Y}-\mathrm{GT}(\mathrm{U} / \mathrm{L})$

CEA(ug/L)

CA19-9(ku/L)

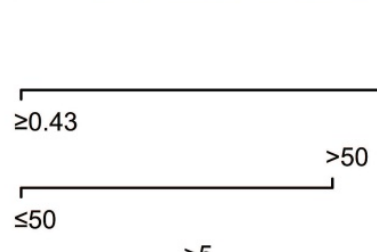

$\leq 5$

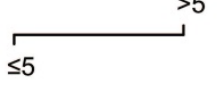

TNM stage
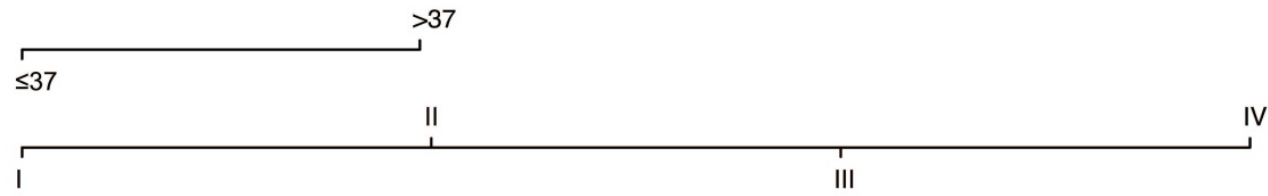

Total Points

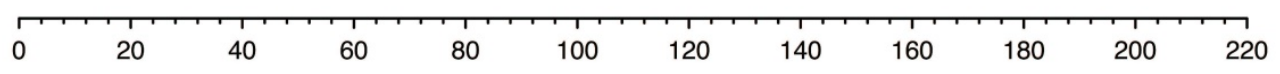

1-year survival

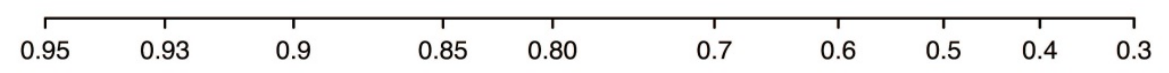

2-year survival

\begin{tabular}{|c|c|c|c|c|c|c|c|c|c|}
\hline 0.85 & 0.80 & 0.7 & 0.6 & 0.5 & 0.4 & 0.3 & 0.2 & 0.1 & 0.05 \\
\hline
\end{tabular}

3-year survival

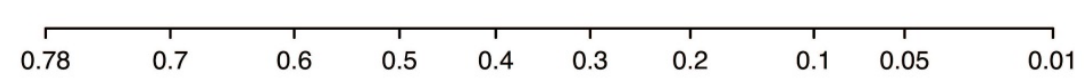

Figure 4. The AAPR-based prognostic nomogram for cHCC-CCA patients. AAPR: albumin-to-alkaline phosphatase ratio; Y-GT: Y-glutamyl transpeptidase; CEA: carcinoembryonic antigen; CA19-9: carbohydrate antigen 19-9.

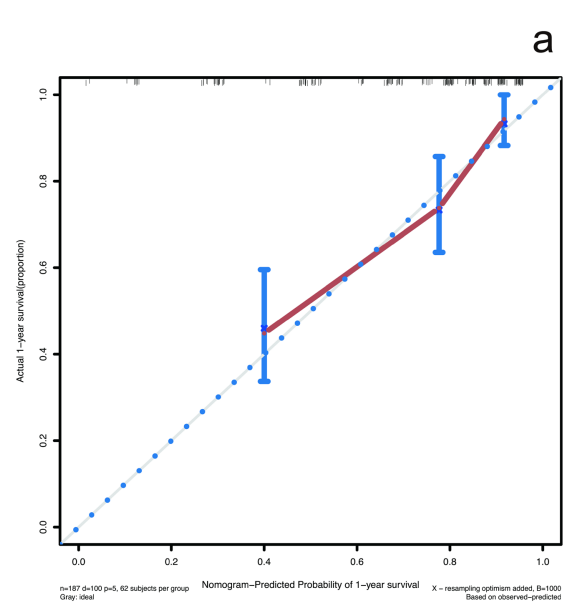

a

d

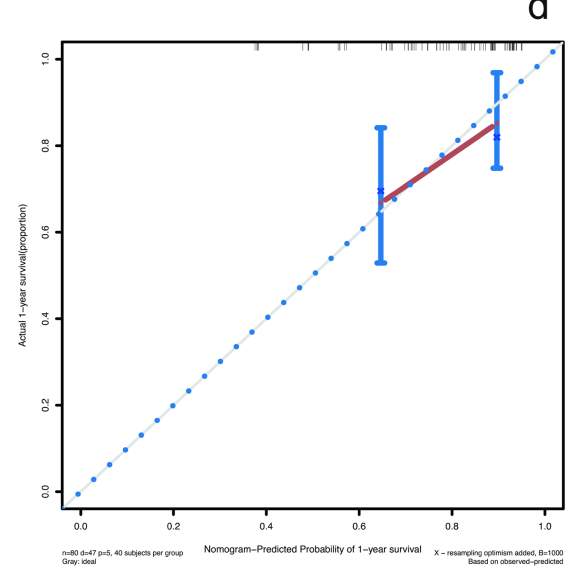

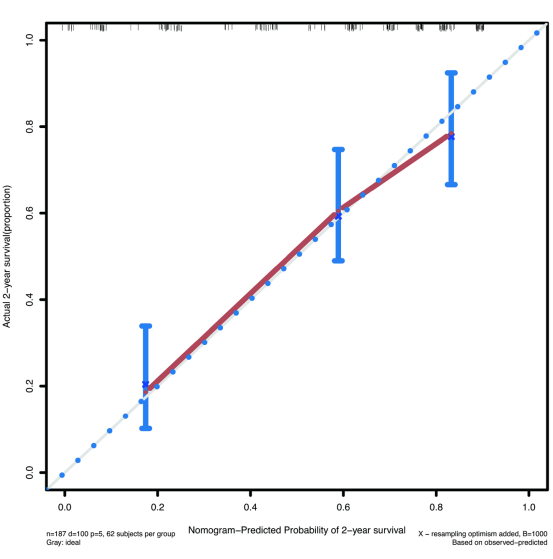

b

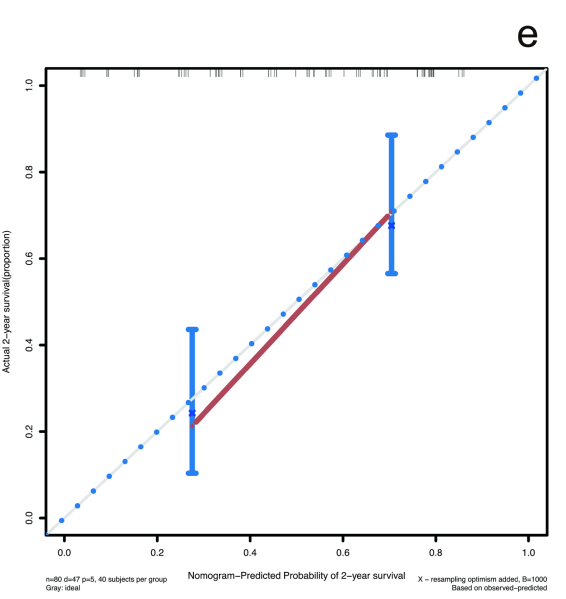

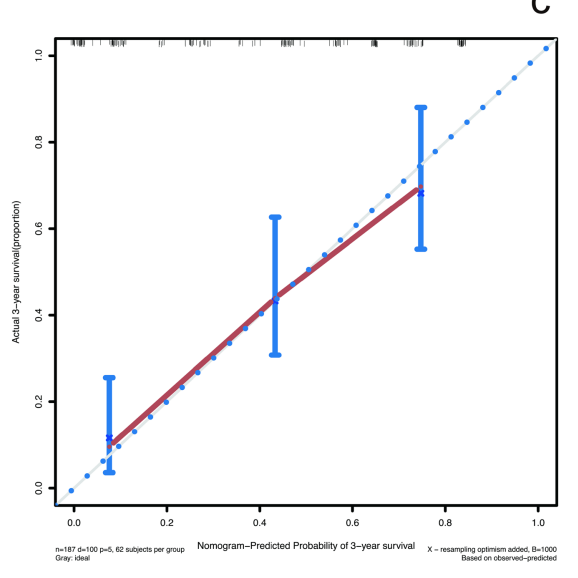

C

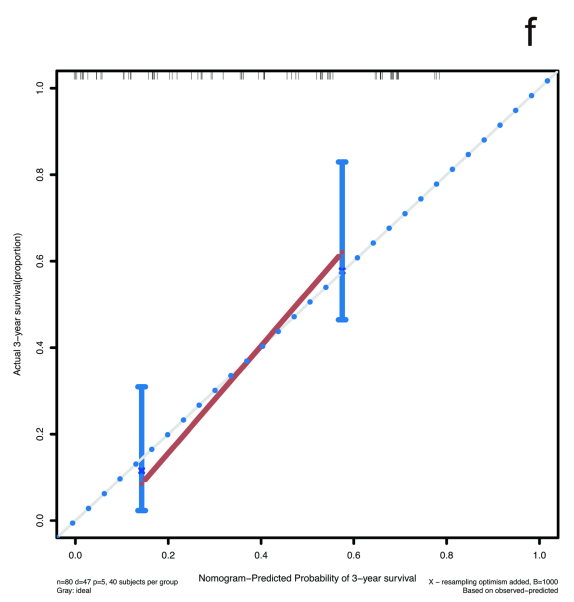

Figure 5. Calibration curves for predicting 1-, 2- and 3-year OS in patients with CHCC-CCA in the training cohort and validation cohort. (a-c) calibration curves for predicting 1-, 2- and 3-year OS in the training cohort; (d-f) calibration curves for predicting 1-, 2-and 3-year OS in the validation cohort. OS: overall survival. 
Table 5. The predictive performances of the AAPR-based prognostic nomogram in comparison with the AJCC TNM-8

\begin{tabular}{|c|c|c|c|c|c|c|c|c|}
\hline \multirow[t]{2}{*}{ Model } & \multicolumn{4}{|l|}{ Training cohort } & \multicolumn{4}{|l|}{ Validation cohort } \\
\hline & C-index $(95 \% \mathrm{CI})$ & AUC $(95 \% \mathrm{CI})$ & LAT $\chi^{2}$ & $\mathrm{AIC}$ & C-index $(95 \% \mathrm{CI})$ & AUC $(95 \% \mathrm{CI})$ & LAT $\chi^{2}$ & AIC \\
\hline Nomogram & $0.76(0.71-0.81)$ & $0.77(0.70-0.83)$ & 65.2 & 800 & $0.69(0.60-0.78)$ & $0.74(0.63-0.86)$ & 27.7 & 281 \\
\hline AJCC TNM-8 & $0.70(0.65-0.75)$ & $0.66(0.58-0.73)$ & 32.0 & 833 & $0.65(0.56-0.74)$ & $0.71(0.61-0.82)$ & 21.4 & 287 \\
\hline
\end{tabular}

AAPR at 0.43 was determined as the optimal cut-off value in present study. cHCC-CCA patients with AAPR $<0.43$ were divided into low-AAPR group, whereas patients with AAPR $\geq 0.43$ were divided into high-AAPR group. Patients with low levels of AAPR had significantly unfavorable prognosis in both training and validation cohorts $(\mathrm{P}<$ $0.001 \& P=0.001$, Figure 1). Moreover, based on univariate and multivariate analyses, AAPR was identified as an independent prognostic factor in the training cohort as well as validation cohort $(\mathrm{P}=0.007$ $\& P=0.034$, Table 3 ). These results collectively indicated that preoperative AAPR was an important prognostic predictor in CHCC-CCA.

ALB and AKP is the two basic parameter incorporated into AAPR, which are easily accessible and relatively inexpensive routine laboratory indices. AKP is a hydrolase enzyme with multiple isoforms that is mainly expressed in liver, bile duct and bone, etc. [15]. It has been proposed that certain pathological conditions including biliary cirrhosis, tumorigenesis of HCC or CC and liver injury may increase AKP levels [21, 22]. Although the prognostic value of AKP has been scarcely discussed in cHCC-CCA, it has been identified as a prognostic indicator for HCC, CC $[23,24]$ and other malignancies $[25,26]$. Regarding the underlying mechanisms, an in vitro study showed that high AKPase activities in the nucleolus may be related to high levels of proliferation of tumor cells [27]. Some researchers also identified the association between AKP and epithelial mesenchymal transition (EMT) in HCC [28, 29]. In addition, it's reported that AKP can be an indicator of oxidative stress, which plays a major role in inflammation [15]. Therefore, in primary liver cancer, AKP is an index which not only associates with liver function, but also links to tumor proliferation, invasion and inflammation status. ALB is an important index in CP grade. It's reported that ALB can predict survival in several malignancies including HCC, CC, colorectal cancer and renal cell carcinoma, etc. [30-32]. Moreover, serum ALB serves as a nutritional index and reflects synthetic function of liver. Low levels of ALB can result in impairment of human immunity and eventually contribute to poor prognosis [33]. Investigations proposed that ALB also modulated inflammatory reaction, which plays a major role in tumorigenesis [34]. In addition, a recent research showed that ALB suppressed the proliferation of HCC cells directly by increasing the G0/G1 cell population [35].

To the best of our knowledge, the CP grade and ALBI grade are the most widely used assessment tools for liver function reverse. Regarding $\mathrm{CP}$ grade, it has been set as an important index in several staging systems including the Barcelona Clinic Liver Cancer (BCLC) staging system [36], the Cancer of the Liver Italian Program (CLIP) score [37] and the Japan integrated staging (JIS) score [38]. However, the CP grade was initially derived from patients with liver cirrhosis, and cirrhosis is not always accompanied with cHCC-CCA [20]. Our previous study has identified that CHCC-CCA is a liver malignancy with lower incidence of cirrhosis in comparison with HCC [39]. Moreover, CP grade incorporates two highly subjective indices of ascites and hepatic encephalopathy, which compromises its prediction performance in liver malignancy. ALBI grade, developed based on a large cohort of HCC patients, is a more objective method for liver function evaluation [40]. Although ALBI grade has showed good performance in HCC patients, its discrimination efficacy in cHCC-CCA has not been confirmed. In present study, unfortunately, both $\mathrm{CP}$ grade and ALBI grade failed to display prognostic values in CHCCCCA based on multivariate analysis. In contrast, AAPR was identified as an independent predictor of prognosis in the training cohort as well as the validation cohort (Table 3). Moreover, the C-index, AUCs, LAT $\chi^{2}$ and AIC demonstrated that AAPR had a better discrimination efficacy in comparison with $\mathrm{CP}$ grade and ALBI grade in cHCC-CCA (Table 4, Figure 2). DCA based on net benefit and threshold possibilities also demonstrated that AAPR may achieve better clinical usefulness compared with $\mathrm{CP}$ grade and ALBI grade (Figure 3 ).

To facilitate prognosis prediction in CHCC-CCA patients, an accurate prognostic model is in utmost need. To date, the most widely accepted prognostic system for CHCC-CCA is the AJCC TNM system [16]. However, TNM system only takes tumor characteristics into account and lacks liver function indices, which compromised its predictive performance in liver malignancies with background 
hepatitis or liver cirrhosis. To address this problem, we constructed a nomogram based on 5 variables including TNM stage, AAPR, Y-GT, CEA and CA19-9 (Figure 4). The AAPR-based prognostic nomogram achieved C-index and AUC values of 0.76 (95\%CI: $0.71-0.81)$ and 0.77 (95\% CI: 0.70-0.83) in the training cohort and of 0.69 (95\% CI: 0.60-0.78) and 0.74 (95\%CI: 0.63-0.86) in the validation cohort, which significantly outperformed the TNM stage. The LAT and AIC values also supported this finding (Table 5).

Surgical resection is the only curative option for localized cHCC-CCA [20]. Several recent studies showed postoperative TACE [41-43] and chemotherapy $[7,44,45]$ may be effective adjuvant treatments for cHCC-CCA undergoing surgical treatment. Therefore, with the AAPR-based nomogram, clinicians can identify patients with poor survival outcome after liver resection. For these patients, the adjuvant treatments such as TACE and chemotherapy are recommended. Moreover, cHCCCCA patients with poor prognosis should be followed up more intensively after surgery.

However, there are some limitations in this study. First, this is a retrospective research conducted in a single center, which may cause selection bias. Second, due to the rarity of cHCC-CCA as well as incomplete records in publicly available databases, external validations were not performed yet. Third, the threshold value of AAPR in present study was determined by the X-tile software, no consensual cut-off value has been proposed yet. Fourth, AAPR should be employed with full consideration of concurrent malignancies, bone and renal diseases.

In summary, our study identifies that the albumin-to-alkaline phosphatase ratio is an independent predictor of prognosis in cHCC-CCA. Based on this finding, we develop an AAPR-based nomogram for prognosis prediction and further decision making in cHCC-CCA. Future external validation studies and prospective researches from different cohorts are still warranted.

\section{Abbreviations}

HR: hazard ratio; $\mathrm{CI}$ : confidence interval; cHCC-CCA: combined hepatocellular and cholangiocarcinoma; HCC: hepatocellular carcinoma; CC: cholangiocarcinoma; HPCs: hepatic progenitor cells; WHO: World Health Organization; CP grade: Child-Pugh grade; CEA: carcinoembryonic antigen; CA19-9: carbohydrate antigen 19-9; AAPR: Albuminto-Alkaline Phosphatase Ratio; ALB: albumin; AKP: alkaline phosphatase; OS: overall survival; CT: computerized tomography; MRI: magnetic resonance imaging; SD: standard deviation; ALBI grade: albumin-bilirubin grade; AUC: area under the curve;
LAT: likelihood ratio test; AIC: Akaike information criteria; C-index: concordance index; DCA: decision curve analysis; HBV: hepatitis B virus; HCV: hepatitis C virus; AFP: Alfa-Fetoprotein; MVI: macroscopic vascular invasion; LNI: lymph node involvement; Y-GT: $\gamma$-glutamyl transpeptidase; ALT: alanine aminotransferase; TB: total bilirubin; EMT: epithelial mesenchymal transition; BCLC: the Barcelona Clinic Liver Cancer staging system; CLIP: the Cancer of the Liver Italian Program score; AJCC TNM-8: the 8th edition of American Joint Committee on Cancer TNM staging.

\section{Supplementary Material}

Supplementary figure S1.

http://www.jcancer.org/v11p5177s1.pdf

\section{Acknowledgements}

\section{Funding}

This work was supported by the National Natural Science Foundation of China [grant number: 81972889] and Excellent Backbone Foundation of Zhongshan hospital, Fudan University [grant number: 2019ZSGG20].

\section{Competing Interests}

The authors have declared that no competing interest exists.

\section{References}

1. Ramai D, Ofosu A, Lai JK, Reddy M, Adler DG. Combined Hepatocellular Cholangiocarcinoma: A Population-Based Retrospective Study. Am J Gastroenterol. 2019; 114: 1496-501.

2. Zhang F, Chen XP, Zhang W, Dong HH, Xiang S, Zhang WG, et al. Combined hepatocellular cholangiocarcinoma originating from hepatic progenitor cells: immunohistochemical and double-fluorescence immunostaining evidence. Histopathology. 2008; 52: 224-32.

3. Allen RA, Lisa JR. Combined liver cell and bile duct carcinoma. The American journal of pathology. 1949; 25: 647-55.

4. Nagtegaal ID, Odze RD, Klimstra D, Paradis V, Rugge M, Schirmacher P, et al. The 2019 WHO classification of tumours of the digestive system. Histopathology. 2019.

5. Lee JH, Chung GE, Yu SJ, Hwang SY, Kim JS, Kim HY, et al. Long-term Prognosis of Combined Hepatocellular and Cholangiocarcinoma After Curative Resection Comparison With Hepatocellular Carcinoma and Cholangiocarcinoma. Journal of Clinical Gastroenterology. 2011; 45: 69-75.

6. Lee SD, Park SJ, Han SS, Kim SH, Kim YK, Lee SA, et al. Clinicopathological features and prognosis of combined hepatocellular carcinoma and cholangiocarcinoma after surgery. Hepatobiliary \& Pancreatic Diseases International. 2014; 13: 594-601.

7. Wakizaka K, Yokoo H, Kamiyama T, Ohira M, Kato K, Fujii Y, et al. Clinical and pathological features of combined hepatocellular-cholangiocarcinoma compared with other liver cancers. Journal of Gastroenterology and Hepatology. 2019; 34: 1074-80.

8. Kobayashi S, Terashima T, Shiba S, Yoshida Y, Yamada I, Iwadou S, et al. Multicenter retrospective analysis of systemic chemotherapy for unresectable combined hepatocellular and cholangiocarcinoma. Cancer Science. 2018; 109: 2549-57.

9. Song P, Midorikawa Y, Nakayama H, Higaki T, Moriguchi M, Aramaki O, et al. Patients' prognosis of intrahepatic cholangiocarcinoma and combined hepatocellular-cholangiocarcinoma after resection. Cancer Medicine. 2019; 8: 5862-71.

10. Ye J, Xie X, Liu B, Zhang X, Wang W, Huang X, et al. Imaging Features on Contrast-Enhanced Ultrasound and Clinical Characteristics of Hepatitis B Virus-Related Combined Hepatocellular-Cholangiocarcinoma: Comparison with Hepatitis B Virus-Related Hepatocellular Carcinoma. Ultrasound in Medicine and Biology. 2017; 43: 2530-6. 
11. Chan AWH, Chan SL, Mo FKF, Wong GLH, Wong VWS, Cheung YS, et al. Albumin-to-Alkaline Phosphatase Ratio: A Novel Prognostic Index for Hepatocellular Carcinoma. Disease Markers. 2015: 1-10.

12. Chen $\mathrm{ZH}$, Zhang XP, Cai XR, Xie SD, Liu MM, Lin JX, et al. The Predictive Value of Albumin-to-Alkaline Phosphatase Ratio for Overall Survival of Hepatocellular Carcinoma Patients Treated with Trans-Catheter Arterial Chemoembolization Therapy. Journal of Cancer. 2018; 9: 3467-78.

13. Xiong JP, Long JY, Xu WY, Bian J, Huang HC, Bai Y, et al. Albumin-to-alkaline phosphatase ratio: A novel prognostic index of overall survival in cholangiocarcinoma patients after surgery. World Journal of Gastrointestinal Oncology. 2019; 11: 39-47.

14. Pu N, Gao S, Xu Y, Zhao G, Lv Y, Nuerxiati A, et al. Alkaline Phosphatase-To-Albumin Ratio as a Prognostic Indicator in Pancreatic Ductal Adenocarcinoma after Curative Resection. Journal of Cancer. 2017; 8: 3362-70.

15. Li SJ, Lv WY, Du H, Li YJ, Zhang WB, Che GW, et al. Albumin-to-alkaline phosphatase ratio as a novel prognostic indicator for patients undergoing minimally invasive lung cancer surgery: Propensity score matching analysis using a prospective database. International Journal of Surgery. 2019; 69: 32-42.

16. Chun YS, Pawlik TM, Vauthey JN. 8th Edition of the AJCC Cancer Staging Manual: Pancreas and Hepatobiliary Cancers. Annals of Surgical Oncology. 2018; 25: 845-7.

17. Sun HC, Tang ZY, Wang L, Qin LX, Ma ZC, Ye QH, et al. Postoperative interferon alpha treatment postponed recurrence and improved overall survival in patients after curative resection of HBV-related hepatocellular carcinoma: a randomized clinical trial. Journal of Cancer Research and Clinical Oncology. 2006; 132: 458-65

18. Camp RL, Dolled-Filhart M, Rimm DL. X-tile: A new bio-informatics tool for biomarker assessment and outcome-based cut-point optimization. Clinical Cancer Research. 2004; 10: 7252-9.

19. Vickers AJ, Elkin EB. Decision curve analysis: A novel method for evaluating prediction models. Med Decis Mak. 2006; 26: 565-74.

20. Komuta M, Yeh MM. A Review on the Update of Combined Hepatocellular Cholangiocarcinoma. Seminars in liver disease. 2019.

21. Giljaca V, Stimac D, Gluud C. Are Levels of Alkaline Phosphatases and Bilirubin Surrogate Markers of Outcomes of Patients With Primary Biliary Cirrhosis? Gastroenterology. 2015; 148: 860-.

22. Al Mamari S, Djordjevic J, Halliday JS, Chapman RW. Improvement of serum alkaline phosphatase to $<1.5$ upper limit of normal predicts better outcome and reduced risk of cholangiocarcinoma in primary sclerosing cholangitis. Journal of Hepatology. 2013; 58: 329-34.

23. Zhang CY, Wang HY, Ning ZY, Xu LIT, Zhuang LP, Wang P, et al. Serum liver enzymes serve as prognostic factors in patients with intrahepatic cholangiocarcinoma. Oncotargets and Therapy. 2017; 10: 1441-9.

24. Sun HC, Xie L, Yang XR, Li W, Yu J, Zhu XD, et al. Shanghai Score: A Prognostic and Adjuvant Treatment-evaluating System Constructed for Chinese Patients with Hepatocellular Carcinoma after Curative Resection. Chinese Medical Journal. 2017; 130: 2650-+.

25. Fernandez-Garcia D, Hills A, Page K, Hastings RK, Toghill B, Goddard KS, et al. Plasma cell-free DNA (cfDNA) as a predictive and prognostic marker in patients with metastatic breast cancer. Breast Cancer Res. 2019; 21: 13.

26. Namikawa T, Ishida N, Tsuda S, Fujisawa K, Munekage E, Iwabu J, et al. Prognostic significance of serum alkaline phosphatase and lactate dehydrogenase levels in patients with unresectable advanced gastric cancer. Gastric Cancer. 2019; 22: 684-91.

27. Yamamoto K, Awogi T, Okuyama K, Takahashi N. Nuclear localization of alkaline phosphatase in cultured human cancer cells. Medical Electron Microscopy. 2003; 36: 47-51.

28. Iwadate $Y$, Matsutani T, Hirono S, Shinozaki N, Saeki N. Transforming growth factor-beta and stem cell markers are highly expressed around necrotic areas in glioblastoma. Journal of Neuro-Oncology. 2016; 129: 101-7.

29. Yi JK, Mehrazarin S, Oh JE, Bhalla A, Oo J, Chen W, et al. Osteo-/Odontogenic Differentiation of Induced Mesenchymal Stem Cells Generated through Epithelial-Mesenchyme Transition of Cultured Human Keratinocytes. Journal of Endodontics. 2014; 40: 1796-801.

30. Nazha B, Moussaly E, Zaarour M, Weerasinghe C, Azab B. Hypoalbuminemia in colorectal cancer prognosis: Nutritional marker or inflammatory surrogate? World Journal of Gastrointestinal Surgery. 2015; 7: 370-7.

31. Chen Z, Shao Y, Fan M, Zhuang Q, Wang K, Cao W, et al. Prognostic significance of preoperative $\mathrm{C}$-reactive protein: albumin ratio in patients with clear cell renal cell carcinoma. International journal of clinical and experimental pathology. 2015; 8: 14893-900.

32. Levillain $\mathrm{H}$, Derijckere ID, Ameye $\mathrm{L}$, Guiot $\mathrm{T}$, Braat $\mathrm{A}$, Meyer $\mathrm{C}$, et al. Personalised radioembolization improves outcomes in refractory intra-hepatic cholangiocarcinoma: a multicenter study. European Journal of Nuclear Medicine and Molecular Imaging. 2019; 46: 2270-9.

33. Tanriverdi O. A discussion of serum albumin level in advanced-stage hepatocellular carcinoma: a medical oncologist's perspective. Medical Oncology. 2014; 31.

34. Arroyo V, Garcia-Martinez R, Salvatella X. Human serum albumin, systemic inflammation, and cirrhosis. Journal of Hepatology. 2014; 61: 396-407.

35. Nojiri S, Joh T. Albumin Suppresses Human Hepatocellular Carcinoma Proliferation and the Cell Cycle. International Journal of Molecular Sciences. 2014; 15: 5163-74.

36. Llovet JM, Bru C, Bruix J. Prognosis of hepatocellular carcinoma: The BCLC staging classification. Seminars in Liver Disease. 1999; 19: 329-38.
37. Manghisi G, Elba S, Mossa A, Giorgio A, Aloisio V, Perrotta A, et al. A new prognostic system for hepatocellular carcinoma: A retrospective study of 435 patients. Hepatology. 1998; 28: 751-5.

38. Kudo M, Chung HB, Osaki Y. Prognostic staging system for hepatocellular carcinoma (CLIP score): its value and limitations, and a proposal for a new staging system, the Japan Integrated Staging Score (JIS score). Journal of Gastroenterology. 2003; 38: 207-15.

39. Yin $X$, Zhang BH, Qiu SJ, Ren ZG, Zhou J, Chen $\mathrm{XH}$, et al. Combined Hepatocellular Carcinoma and Cholangiocarcinoma: Clinical Features, Treatment Modalities, and Prognosis. Annals of Surgical Oncology. 2012; 19: 2869-76.

40. Johnson PJ, Berhane S, Kagebayashi C, Satomura S, Teng M, Reeves HL, et al. Assessment of Liver Function in Patients With Hepatocellular Carcinoma: A New Evidence-Based Approach-The ALBI Grade. Journal of Clinical Oncology. 2015; 33: 550-U45.

41. Morizono S, Fukutomi M, Yokota M, Iguchi H, Funakoshi A, Wakasugi H, et al. A case of combined liver cell and bile duct carcinoma detected 6 years after interferon therapy for chronic hepatitis $\mathrm{C}$, in spite of showing complete response. Japanese Journal of Gastroenterology. 2002; 99: 505-10.

42. Hayashi H, Beppu T, Ishiko T, Mizumoto T, Masuda T, Okabe K, et al. A 42-month disease free survival case of combined hepatocellular-cholangiocarcinoma with lymph node metastases treated with multimodal therapy. Gan to kagaku ryoho Cancer \& chemotherapy. 2006; 33: 1941-3.

43. Tani S, Murata S, Tamura M, Fukunaga K, Morita M, Hirata Y, et al. Effectiveness of systemic chemotherapy of GEM+CBDCA+5-FU/LV and hepatic arterial infusion of CDDP in a case of advanced, combined hepatocellular-cholangiocarcinoma with multiple lung metastases. Nihon Shokakibyo Gakkai zasshi = The Japanese journal of gastro-enterology. 2011; 108: 1892-901.

44. Kim GM, Jeung H-C, Kim D, Kim JH, Yoon SH, Jung ES, et al A Case of Combined Hepatocellular-Cholangiocarcinoma with Favorable Response to Systemic Chemotherapy. Cancer Research and Treatment. 2010; 42: 235-8.

45. Chi M, Mikhitarian K, Shi C, Goff LW. Management of combined hepatocellular-cholangiocarcinoma: a case report and literature review. Gastrointestinal cancer research : GCR. 2012; 5: 199-202. 